\title{
Regulation of Experience-Dependent Bidirectional Chemotaxis by a Neural Circuit Switch in Caenorhabditis elegans
}

\author{
Yohsuke Satoh, ${ }^{1}$ Hirofumi Sato, ${ }^{1}$ Hirofumi Kunitomo, ${ }^{1}$ Xianfeng Fei, ${ }^{2}$ Koichi Hashimoto, ${ }^{3}$ and Yuichi Iino ${ }^{1,4}$ \\ ${ }^{1}$ Department of Biological Sciences, Graduate School of Science, University of Tokyo, Bunkyo-ku, Tokyo 113-0033, Japan, ${ }^{2}$ Department of Intelligent \\ Information Systems, Faculty of Science and Technology, Tohoku Bunka Gakuen University, Aoba-ku, Sendai 980-8579, Japan, ${ }^{3}$ Department of System \\ Information Sciences, Graduate School of Information Sciences, Tohoku University, Aoba-ku, Sendai 980-8579, Japan, and ${ }^{4}$ CREST, Japan Science and \\ Technology Agency, Kawaguchi, Saitama 332-0012, Japan
}

The nematode Caenorhabditis elegans changes its chemotaxis to $\mathrm{NaCl}$ depending on previous experience. At the behavioral level, this chemotactic plasticity is generated by reversing the elementary behaviors for chemotaxis, klinotaxis, and klinokinesis. Here, we report that bidirectional klinotaxis is achieved by the proper use of at least two different neural subcircuits. We simulated an NaCl concentration change by activating an NaCl-sensitive chemosensory neuron in phase with head swing and successfully induced klinotaxis-like curving. The curving direction reversed depending on preconditioning, which was consistent with klinotaxis plasticity under a real concentration gradient. Cell-specific ablation and activation of downstream interneurons revealed that ASER-evoked curving toward lower concentration was mediated by AIY interneurons, whereas curving to the opposite direction was not. These results suggest that the experiencedependent bidirectionality of klinotaxis is generated by a switch between different neural subcircuits downstream of the chemosensory neuron.

Key words: C. elegans; chemotaxis; klinotaxis; plasticity

\section{Introduction}

Behaviors, which are the prominent outputs of the nervous system, are often generated in response to sensory inputs. Although the transformation of information in this process is a major focus of neuroscience, it is difficult to figure out exactly how behavioral outputs are generated in higher animals. In the soil nematode Caenorhabditis elegans, all 302 neurons and their connections have been mapped completely (White et al., 1986), and C. elegans chemosensation and chemotactic behaviors have been well described (Ward, 1973; Bargmann and Horvitz, 1991). This understanding of the nematode's nervous system offers advantages for studying the processes between inputs and outputs at a cellular resolution by using the single-neuron manipulation techniques that are available for this model organism.

For chemotaxis, the relationships between chemicals as inputs and locomotion as outputs are well studied. As one of the two major behavioral strategies for chemotaxis, C. elegans employs

\footnotetext{
Received May 1, 2014; revised Sept. 8, 2014; accepted Oct. 4, 2014.

Author contributions: Y.I. designed research; Y.S. performed research; H.S., H.K., X.F., and K.H. contributed unpublished reagents/analytic tools; Y.S. analyzed data; Y.S. wrote the paper.

This work was supported by Grants-in-Aid for Innovative Areas Systems Molecular Ethology and Memory Dynamics to Y.I. and Mesoscopic Neurocircuitry to H.K. We also thank HawkVision and Olympus for their help in setting up the tracking system used in this research.

The authors declare no competing financial interests.

Correspondence should be addressed to Yuichi lino, Science Building \#3, Room 224, 7-3-1 Hongo, Bunkyo-ku, Tokyo 113-0033, Japan. E-mail: iino@bs.s.u-tokyo.ac.jp.

DOI:10.1523/JNEUROSCI.1757-14.2014

Copyright $\odot 2014$ the authors $\quad 0270-6474 / 14 / 3315631-07 \$ 15.00 / 0$
}

klinotaxis; when the animal faces a concentration gradient perpendicular to its body axis, it gradually curves toward the concentration peak of the chemical (Fig. 1a; Iino and Yoshida, 2009). Course correction in klinotaxis is thought to be exerted by biasing motor outputs during sinusoidal forward movement in response to the chemical gradient. The gradient is probably detected as changes in the chemical input caused by sinusoidal movements of the head, where the chemosensing amphid sensilla are located (Izquierdo and Lockery, 2010). This view has been supported by a set of experiments in which the optogenetic activation or inactivation of the odor-sensitive AWC ${ }^{\mathrm{ON}}$ neuron in synchrony with the animal's head swing induced curving outputs that corresponded to positive klinotaxis to odor (Fig. 1b; Kocabas et al., 2012).

Unlike klinotaxis to odor, klinotaxis to $\mathrm{NaCl}$ is bidirectional, and it changes as a result of learning. C. elegans memorizes the $\mathrm{NaCl}$ concentration at which it is cultivated with food and in a following test it shows chemotaxis to the memorized $\mathrm{NaCl}$ concentration. Thus, klinotaxis to $\mathrm{NaCl}$ can be either positive or negative, depending on the difference between the memorized concentration and the concentration at the position of the worm. Klinotaxis in both directions requires inputs from a single chemosensory neuron, ASER, which is activated in response to the decrease in $\mathrm{NaCl}$ concentration (Kunitomo et al., 2013). Although klinotaxis to odor and $\mathrm{NaCl}$ are mediated by different chemosensory neurons (AWC ${ }^{\mathrm{ON}}$ and ASER, respectively), the postsynaptic interneuron classes for these neurons are common: 
AIA, AIB, and AIY. We focused on this commonality of circuits and bidirectionality of the klinotactic outputs to elucidate the neural mechanisms of klinotaxis and its plasticity. We first controlled the activity of ASER neuron in animals conditioned by different $\mathrm{NaCl}$ concentrations. We found that the same activation pattern of a single chemosensory neuron, ASER, generated two opposite curving outputs in correspondence with plastic klinotaxis. We next examined the involvement of interneurons postsynaptic to the ASER neuron by ablation or activation experiments and found that AIY interneurons were required for negative klinotaxis to $\mathrm{NaCl}$ but not for positive klinotaxis. These results suggest that $C$. elegans realizes bidirectional and plastic klinotaxis by the proper use of at least two neural subcircuits: one that contains AIY interneurons and others that do not contain AIY.

\section{Materials and Methods}

Strains and culture. C. elegans strains were cultured and maintained using the standard procedure (Brenner, 1974) with two exceptions. First, NA22 instead of OP50 strain of Escherichia coli was used as a food source to avoid starvation after overcrowding. Second, $C$. elegans SRS281 and SRS291 strains (see below) were cultured and maintained at $25^{\circ} \mathrm{C}$ to select transgenic animals as described previously (Granato et al., 1994; Kocabas et al., 2012). All C. elegans strains were derived from the wildtype strain Bristol N2. The following strains have been made by Kocabas et al. (2012) and were obtained from the Caenorhabditis Genetics Center (University of Minnesota): pha-1(e2123) III; lite-1(ce314) X; sraEx281 $[$ ttx-3p::chop-2(H134R)::TagRFP + pBX(pha1(+))] (SRS281), pha-1(e2123) III; lite1(ce314) X; sraEx291[npr-9p::chop-2(H134R):: TagRFP + pBX(pha-1(+))] (SRS291). The following strains have been made by Kunitomo et al. (2013): lite-1 (ce314) X; peIs1091 [gcy-5p::chop-2 (H134R)::yfp unc-122p::mCherry] (JN1601), lite-1(ce314) X; peIs1091; peIs578[npr-9p::casp1 npr-9p::venus unc-122p::mCherry] (JN1602). The other strains were generated in this study: lite-1(ce314) X; peIs1091; peIs579[ttx-3p::casp1 ttx-3p::venus lin-44p::gfp] (JN1603), lite-1(ce314) X; peIs1091; peIs580[ins-1(short)p::casp1 ins-1(short)p::venus unc-122p:: gfp] (JN1604), lite-1(ce314) X; peEx1605[ins-1(short)p::chop-2(H134R):: yfp unc-122p::gfp] (JN1605), lite-1(ce314) X; peEx1608 [ins-1(short) p::chop-2(H134R)::yfp npr-9p::chop-2(H134R)::yfp unc-122p::mCherry] (JN1608), lite-1(ce314) X; peIs1091; peIs578; peIs580 (JN1607), lite1(ce314) X; peIs1091; peIs579 peIs580 (JN1606). All strains carry the lite1(ce314) mutation to abolish innate avoidance of blue light (Edwards et al., 2008; Liu et al., 2010).

Generation of strains. Transgenic strains were generated by microinjection of transgene DNA to hermaphrodite germline (Mello et al., 1991). Plasmids for germline transformation were generated by the Gateway system (Invitrogen), in which the promoter sequence on an entry vector and the ORF of the gene of interest on a destination vector were combined by LR reaction. Details of this method have been described previously (Matsuki et al., 2006). pENTR-ttx3p, pENTR-ins1(short)p, pDEST-casp1, and pDEST-chop-2(H134R):::yfp have been made previously (Matsuki et al., 2006; Lin et al., 2010; Yoshida et al., 2012; Kunitomo et al., 2013). The AIY-ablated strain peIs579 (JN579) was generated Methods for a description. b

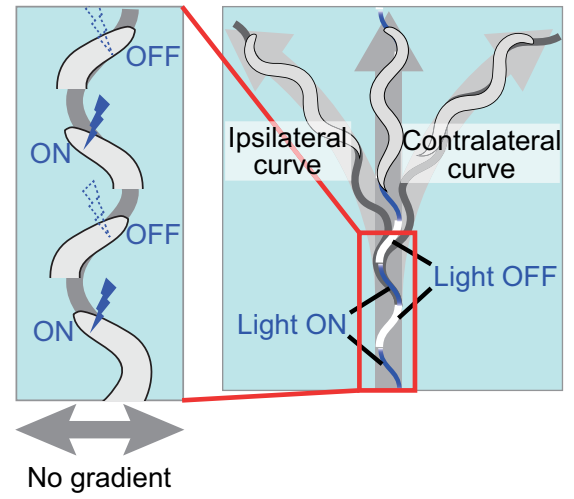

C

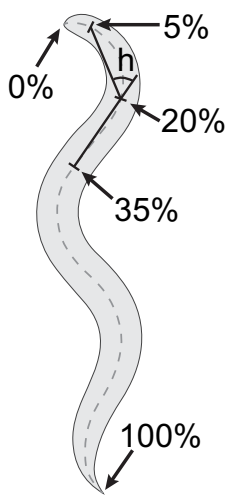

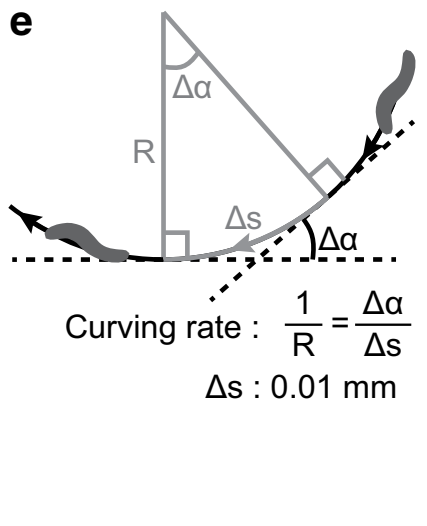

Figure 1. Procedure for optogenetic stimulation of neurons that induce klinotaxis-like curving. $\boldsymbol{a}$, Schematic diagram of klinotaxis. Worms gradually curve toward the side with higher (or lower) chemical concentrations. $\boldsymbol{b}$, Schematic diagram of the . Whe ivation pattern. In the illustration, the animal is stimulated when it bends its neck to the left side. The left curve is ipsilation stimuli. The right curve is contralateral to the stimuli. Quatication of head swing. Neck angle $h$ is defined as

by integrating the transgenes Ex[ttx-3p::casp1] (100 ng/ $\mu \mathrm{l})$ and Ex[ttx-3p::venus] $(100 \mathrm{ng} / \mu \mathrm{l})$ into the genome by UV irradiation followed by outcrossing with N2 three times. For the AIA-ablated strain peIs580 (JN580), the ins-1(short) promoter was used. AIY and AIA neurons were absent in $>90 \%$ of JN579 and JN580 animals, respectively. JN1603, JN1604, JN1606, and JN1607 strains were generated by crossing JN1601 and JN579, JN1601 and JN580, JN1603 and JN1604, and JN1602 and JN1604, respectively. For the JN1605 strain that expresses channelrhodopsin-2 ChR2(H134R) in AIA neurons, Ex[ins-1(short)p::chop-2 (H134R)::yfp] (90 ng/ $\mu \mathrm{l})$ was injected into lite-1(ce314) animals with a coinjection marker. For the JN1608 strain that expresses ChR2(H134R) in AIA and AIB neurons, Ex[ins-1(short)p::chop-2(H134R)::yfp] (50 ng/ $\mu \mathrm{l})$ and Ex[npr-9p::chop-2(H134R)::yfp] (50 ng/ $\mu \mathrm{l})$ were used.

Automatic tracking and stimulation of freely moving nematode. We used an upright microscope (BX51, Olympus) equipped with a halogen light source (U-LH100IR, Olympus), a motorized stage (HV-STU02-1, HawkVision), a CCD camera (GRAS-03K2M-C, Point Grey Research), and a blue LED (470 nm; pE-100, CoolLED) for the worm tracking and stimulation system (Fig. 1d). The animal's centroid, tail, head, and midline were detected and used for tracking and automated blue-light illumination. Tail and head were detected essentially as the sharpest and the second sharpest parts of the body, respectively. Midline was calculated based on the tail position, the head position, and the outline of the animal. The neck angle was defined as the angle between two line seg- 
ments, one between two points at 5 and 20\% of the head-to-tail length along the midline and the other between 20 and $35 \%$ points (Fig. $1 c, \mathrm{~h}$ ). When the neck angle became $>10^{\circ}$ ( or $\left.<-10^{\circ}\right)$, the animal was assumed to be bending its neck to one side and was stimulated by irradiating 0.8 $\mathrm{mW} / \mathrm{mm}^{2}$ of blue light (Fig. $1 b$ ). It took $<20 \mathrm{~ms}$ from image capture to posture detection, and the whole process was performed at $20 \mathrm{~Hz}$ by a custom-made operating software. The software was developed in $\mathrm{C}++$ with OpenCV library and ran on Realtime Linux. During the assay, the worm tracking and stimulation system recorded outline, midline, and location of the animal in a custom format in every $50 \mathrm{~ms}$.

Preculture and assays. All animals used in this study were hermaphrodites. After cultivating on the nematode growth medium (NGM) plates under the standard culture conditions for $4 \mathrm{~d}$, animals at the L4 stage were moved to preconditioning plates and further cultivated at $20^{\circ} \mathrm{C}$ overnight. The preconditioning plates were standard NGM plates, except that their $\mathrm{NaCl}$ concentrations were 25 or $75 \mathrm{~mm}$, and the food source on those plates contained optional $1 \mathrm{~mm}$ all trans-Retinal (ATR; SigmaAldrich), which is necessary for channelrhodopsin to work in C. elegans. Before the assay, each animal was washed with wash buffer (25 mm potassium phosphate, $\mathrm{pH} 6.0,1 \mathrm{~mm} \mathrm{CaCl}_{2}, 1 \mathrm{~mm} \mathrm{MgSO}_{4}, 50 \mathrm{~mm} \mathrm{NaCl}$ ) three times, and was placed on an assay plate. Assay plates were $8.5 \mathrm{~cm}$ in diameter and included $2 \%$ agar in the same ingredients as wash buffer. In each experiment, one animal was tested on an assay plate for $11 \mathrm{~min}$ under the automatic tracking and stimulation system. The animal was allowed to move freely without blue-light stimulation for the first $1 \mathrm{~min}$ and with the stimulation for the remaining $10 \mathrm{~min}$. The stimulation was dependent on the animal's head swing; namely, the blue light was illuminated while the animal's neck was bent on one side, and no stimulation while on the other side (Fig. $1 b$ ). Tracking data were recorded during the assay and for extra $10 \mathrm{~s}$ (670 s in total).

Analysis of locomotion. Stops, omega turns, and backward movements were automatically detected from the recorded midline data and centroid location data. Time periods within $3.18 \mathrm{~s}$ from these events were classified as "not-run" periods, and the remaining periods were classified as "run" periods (adopted from Iino and Yoshida, 2009). If the change in moving direction is $\Delta \alpha$ during the interval $\Delta \mathrm{s}$, then the curving rate is $\Delta \alpha / \Delta \mathrm{s}$ (Fig. 1e). We calculated the curving rate for every $0.01 \mathrm{~mm}$ of run parts. We calculated the average curving rate of an animal only if the total length of the run part of its track was $\geq 10 \mathrm{~mm}$ during the $10 \mathrm{~min}$ test period with stimulation; otherwise the record was discarded. The sign of curving rate was determined so that the value was positive when the animal curved toward the side of the stimulus (ipsilateral side) and the value was negative when the animal curved toward the opposite side (contralateral side). The above criteria were adjusted from Iino and Yoshida (2009) to suit the recorded locomotion data.

\section{Results}

\section{Closed-loop photostimulation of identified neurons}

To achieve photostimulation in phase with head swing, we performed high-speed tracking of moving animals based on the bright-field image of the animals. At the same time, the posture of the animal was extracted by determining the midline of the body. The calculated head-swing angle was used to determine the timing of the blue-light illumination (Fig. $1 b, c, d$; Materials and Methods), thus allowing us to stimulate ChR2-expressing neurons only when the worm swung the head to one side.

\section{The head swing-dependent activation of the ASER neuron causes experience-dependent biased curving}

It was previously reported that a phasic optical stimulation of the odor-sensitive AWC ${ }^{\mathrm{ON}}$ chemosensory neuron using a protocol similar to the one described above made the animals turn away from the direction in which the head was bending when the blue light was turned on ("contralateral curving" to phasic stimulation; Fig. 1b; Kocabas et al., 2012). This activation pattern corresponds to a virtual odor gradient perpendicular to the direction of forward locomotion, and the contralateral curving to phasic activation corresponds to the positive klinotaxis to odor, because the $\mathrm{AWC}^{\mathrm{ON}}$ neuron is activated when odor concentration decreases (Chalasani et al., 2007). We examined whether the same activation pattern of $\mathrm{NaCl}$-sensitive ASER chemosensory neuron generated similar behavioral outputs by using animals that expressed ChR2 only in the ASER neuron (Fig. 1b). To test the effect of $\mathrm{NaCl}$ preference change caused by the difference in previous culture conditions, we precultured the animals with different concentrations of $\mathrm{NaCl}(25,50$, or $75 \mathrm{~mm}$; Fig. $2 a)$ and photostimulated the ASER neuron only when the animal bent its head to one side. In a reaction similar to that with $\mathrm{AWC}^{\mathrm{ON}}$ stimulation, animals curved in response to phasic ASER activation. We found that the curving responses were significantly different between the cases where photostimulation was applied during ventral bending of the head and the cases where it was applied during dorsal bending (Fig. 2b, bottom). This difference was also observed in animals precultivated without ATR, which is essential for photoactivation of ChR2, suggesting that C. elegans has an intrinsic bias toward ventral curving, probably due to dorsoventral asymmetry of the nervous system, and the curving responses to phasic stimulation of ASER also inherits this bias. Considering this, we hereafter conducted the experiments without discriminating the ventral/dorsal stimulation. Apart from the ventral bias observed in all conditions, the animals showed significantly different curving responses to phasic stimulation, depending on preculture conditions; the curving was strongly ipsilateral to the stimulated side when the animals were precultivated at $25 \mathrm{~mm}$, a little contralateral when precultivated at $50 \mathrm{~mm}$, and strongly contralateral when precultivated at $75 \mathrm{~mm}$ (Fig. $2 b$, top, bottom). Knowing that the ASER neuron shows calcium responses to decrease in $\mathrm{NaCl}$ concentration regardless of the $\mathrm{NaCl}$ concentration of precultivation (Kunitomo et al., 2013), we concluded that the ipsilateral curving to the phasic activation of ASER corresponds to the negative klinotaxis to $\mathrm{NaCl}$, and the contralateral curving corresponds to positive klinotaxis. These results were consistent with the previously reported plastic klinotaxis to $\mathrm{NaCl}$ (Kunitomo et al., 2013), in which animals cultivated at a lower concentration curve toward lower $\mathrm{NaCl}$ concentrations and vice versa.

\section{AIY neurons are required for ipsilateral curving to the phasic activation of ASER neuron but not for contralateral curving}

We next examined the involvement of postsynaptic interneurons in the ipsilateral/contralateral curving outputs. The ASER neuron sends synaptic outputs to three pairs of interneurons: AIA, AIB, and AIY (Fig. 3a). Of these, the ablation of AIY interneurons by the cell-specific expression of mouse caspase abolished the ipsilateral curving to the phasic activation of the ASER neuron in animals cultivated at $25 \mathrm{~mm} \mathrm{NaCl}$, but did not affect the contralateral curving of animals cultivated at $75 \mathrm{~mm} \mathrm{NaCl}$ (Fig. $3 b$ ). Ablation of the AIA or AIB interneurons caused little or no defects in both the ipsilateral and contralateral curving (Fig. 3b). However, double ablation of AIA and AIB neurons caused a significant decrease in curving bias on both sides, suggesting that these neurons work redundantly. Effects of AIA/AIY ablation could not be assessed because these animals reversed at an unusually high rate and showed very short run lengths (run length during $10 \mathrm{~min}$ of wild-type JN1601 conditioned with $25 \mathrm{~mm}$ $\mathrm{NaCl}$ without ATR: $43.3 \pm 2.89 \mathrm{~mm}, n=34$; AIA/AIY-ablated strain JN1606: $2.80 \pm 0.54 \mathrm{~mm}, n=31$ ), which was consistent with previous reports that both AIA and AIY neurons suppress reversals (Wakabayashi et al., 2004; Gray et al., 2005). 
a

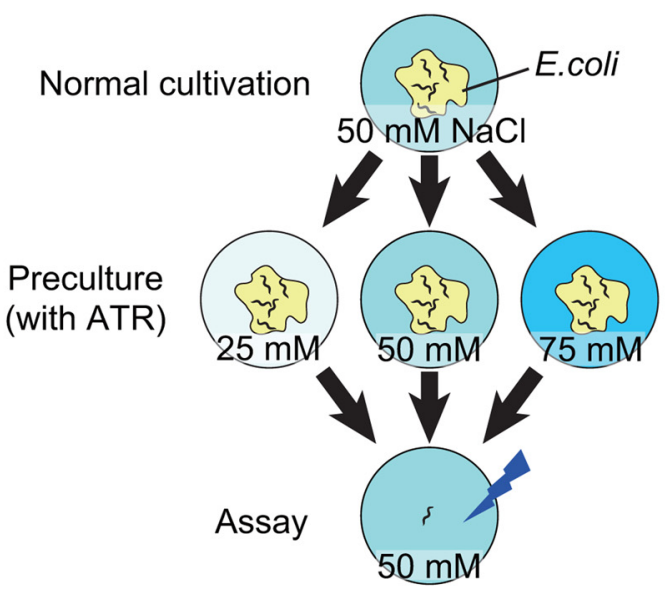

b

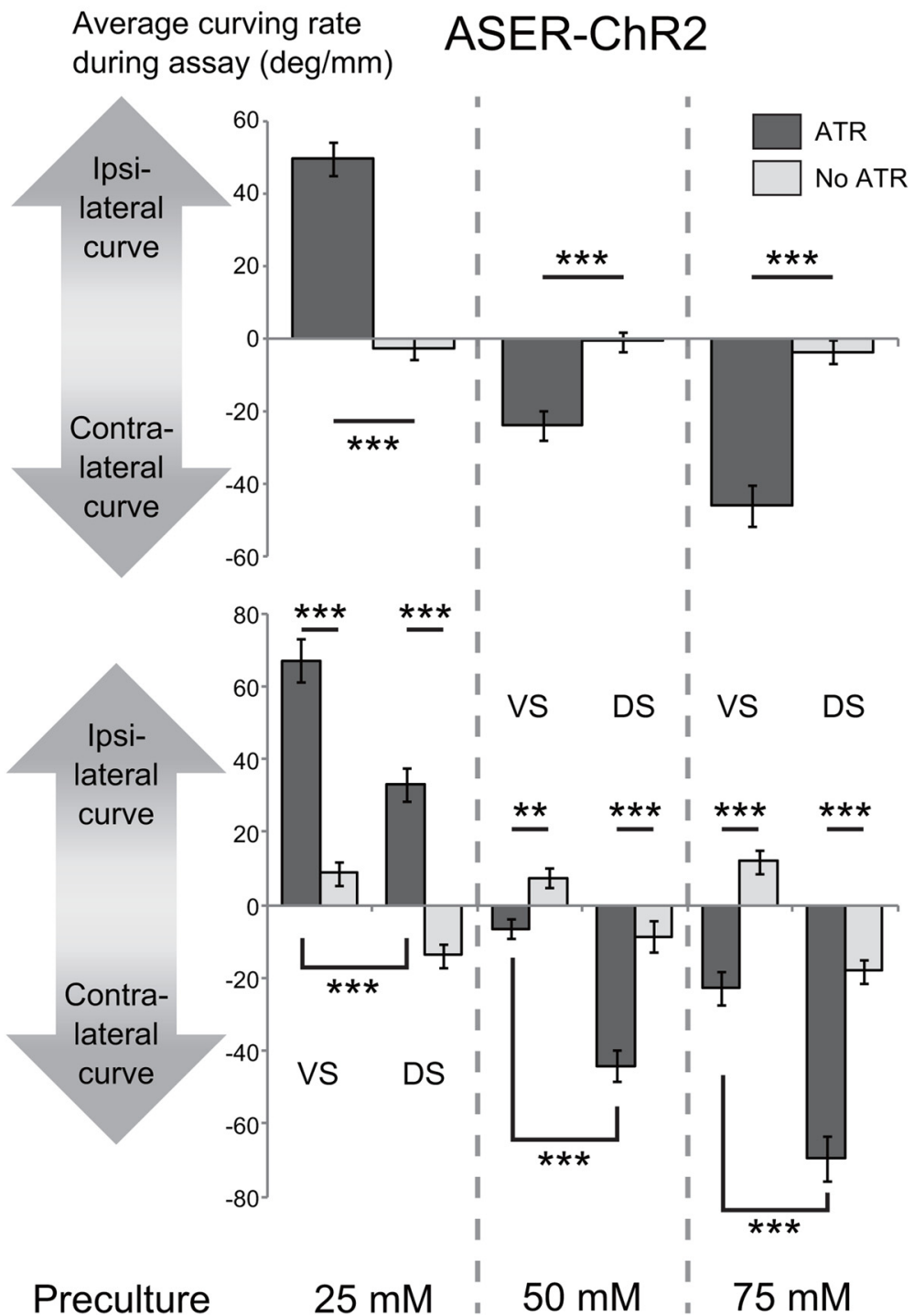

Figure 2. Bidirectional klinotaxis-like curving induced by ASER activation. $\boldsymbol{a}$, Culture and assay conditions. L4 animals were transferred to the preculture plates that contained ATR necessary for channelrhodopsin to work in C. elegans and different concentrations of $\mathrm{NaCl}(25,50$, or $75 \mathrm{~mm})$, and were tested on the next day on the assay plate that contained $50 \mathrm{~mm} \mathrm{NaCl}$. $\boldsymbol{b}$, Curving induced by phasic activation of the ASER neuron after preculture at different concentrations of $\mathrm{NaCl}$. Average curving rate of each animal during the 10 min test period was calculated and the values were averaged across animals that moved forward for $\geq 10 \mathrm{~mm}$ during the 10 min period. In this assay, we recorded whether the side of stimulation was the ventral side or the dorsal side of each animal. "No ATR" indicates the results of the animals precultured without ATR. JN1601 strain was used. Top, Average curving rate during the $10 \mathrm{~min}$ calculated without considering ventral/dorsal difference. The numbers of animals for each bar from left to right were $40,40,37,40,32$, and 39 . The effect of concentration of preculture on curving bias was significant $\left(F_{(2,222)}=80.3, p<2 \times\right.$
Head swing-dependent activation of AIY neurons always causes ipsilateral curving

We next expressed ChR2 in each of the three interneuron classes and examined the effects of optogenetic activation. Direct activation of AIY neurons with the same phasic pattern induced strong ipsilateral curving toward activation stimuli regardless of the $\mathrm{NaCl}$ concentration of cultivation (Fig. 3c, top). Direct phasic activation of AIA neurons generated a weak ipsilateral curving bias when the animals were cultivated at 25 and $50 \mathrm{~mm} \mathrm{NaCl}$, while no significant bias was detected after preincubation at $75 \mathrm{~mm} \mathrm{NaCl}$ (Fig. 3c). Direct phasic activation of AIB neurons alone or AIA and AIB neurons together did not induce any significant bias on curving (Fig. 3c). These results were consistent with the previous proposal that AIY neurons are key neurons that directly regulate ipsilateral curving to the phasic activation of upstream chemosensory neurons (Kocabas et al., 2012). However, AIY neurons cannot generate contralateral curving or contribute to contralateral curving as judged by the cell-ablation experiments. The remaining two interneuron classes, AIA and AIB, do not seem to be able to generate curving bias, even if activated together (Fig. 3c).

\footnotetext{
$\leftarrow$

$\left.10^{-16}\right)$ and the effect of interaction between presence of ATR and concentration of preculture was significant $\left(F_{(2,222)}=\right.$ $85.6, p<2 \times 10^{-16}, 2$-way ANOVA). $p$ values of the statistical test between "ATR" and "no ATR" in each preculture condition were $p=4.55 \times 10^{-15}, p=6.02 \times 10^{-6}$, and $p=2.92 \times 10^{-9}\left({ }^{* * *} p<0.001\right.$, Student's $t$ test). Bottom, Average curving rate in response to ventral stimuli and dorsal stimuli, calculated by the same dataset as the top panel. VS, Ventral stimuli; DS, dorsal stimuli. The numbers of animals for each bar were 20,20,20,20,20,20,17,20,16, 19, 16, and 20 . The main effects or interactions of three factors [presence of ATR while precultivation (ATR), concentration of preculture (concentration), dorsal/ventral difference of stimulation (D/ V)] on curving bias were tested by three-way ANOVA: the effect of concentration was significant $\left(\mathrm{F}_{(2,216)}=146, p<\right.$ $\left.2.2 \times 10^{-16}\right)$; the effect of $\mathrm{D} / \mathrm{V}$ was significant $\left(\mathrm{F}_{(1,216)}=\right.$ $\left.165, p<2.2 \times 10^{-16}\right)$; the effect of interaction between concentration and $\mathrm{D} / \mathrm{V}$ was not significant $\left(F_{(2,216)}=2.31\right.$, $p=0.102)$; the effect of interaction between concentration and ATR was significant $\left(F_{(2,216)}=158, p<2.2 \times 10^{-16}\right)$; and the effect of interaction between $D / V$ and ATR was significant $\left(F_{(1,216)}=11.8, p=7.25 \times 10^{-4}\right)$. Corrected $p$ values $(\mathrm{kp})$ : VS(ATR) versus VS(No ATR), $3.00 \times 10^{-10}, 1.87 \times$ $10^{-9}, 4.16 \times 10^{-4}(25 \mathrm{~mm}$ preculture condition); DS(ATR) versus DS(No ATR), $1.35 \times 10^{-3}, 4.54 \times 10^{-6}, 1.94 \times$ $10^{-8}(50 \mathrm{~mm}$ preculture condition); VS(ATR) versus DS(ATR), $9.66 \times 10^{-7}, 1.01 \times 10^{-8}, 1.87 \times 10^{-9}(75 \mathrm{~mm}$ preculture condition); $k=3$; ${ }^{* * *} k p<0.001,{ }^{* *} k p<0.01,{ }^{*} k p<$ $0.05, t$ test with Bonferroni's correction.
} 

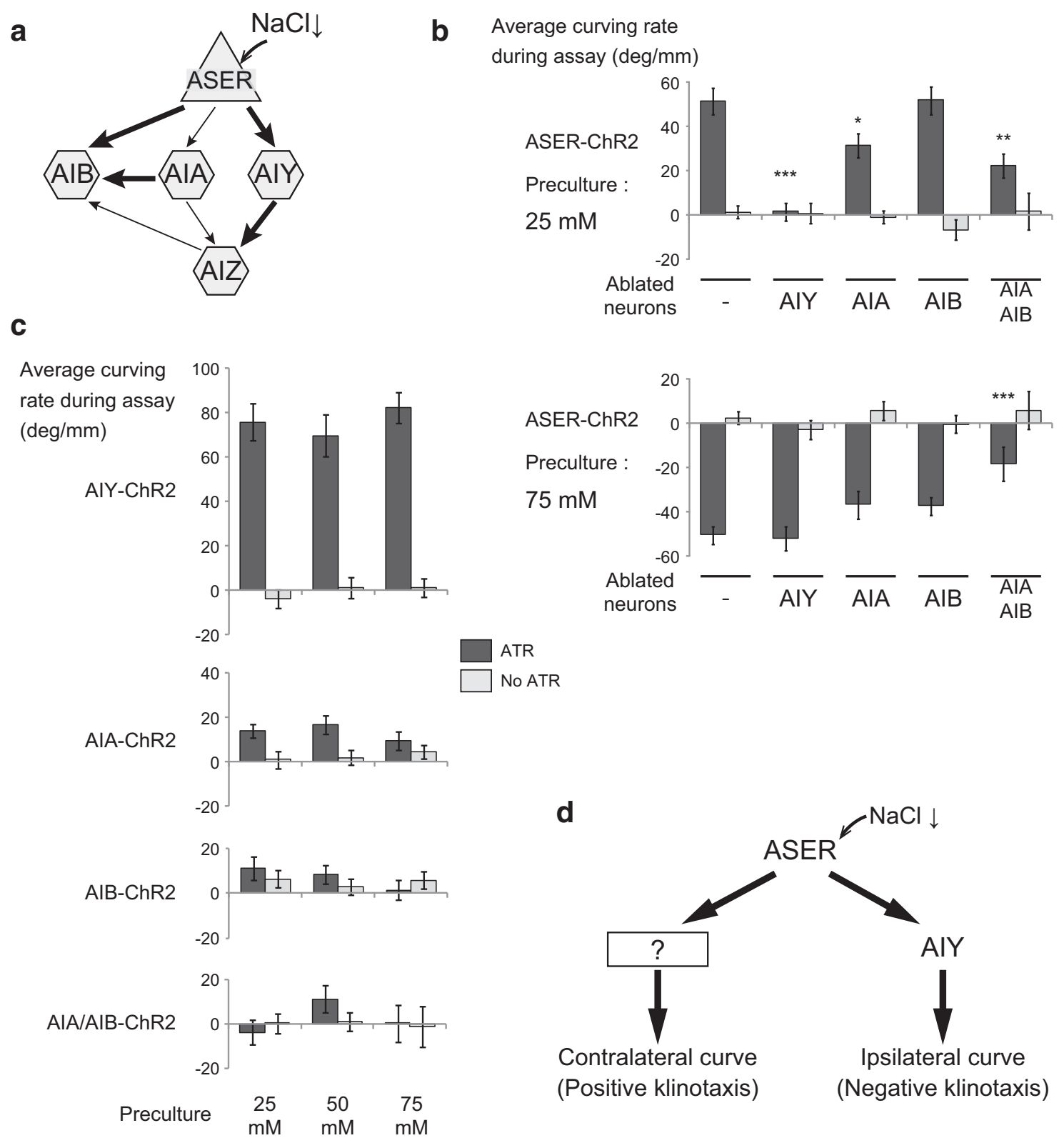

Figure 3. Contribution of downstream interneurons to the two opposite curving outputs. $\boldsymbol{a}$, A wiring diagram of the ASER neuron and the downstream first-layer interneurons. The arrows indicate the synaptic connections, and the thickness of the arrows indicates the number of synapses between the neurons. $\boldsymbol{b}$, Curving induced by phasic activation of the ASER neuron after preculture at either 25 (top) or $75 \mathrm{~mm}$ (bottom) in animals whose interneurons were ablated. JN1601 was used as control and JN1603, JN1604, JN1602, and JN1607 were used as interneuron-ablated strains. The numbers of animals for each bar were (top) 35, 34, 36, 30, 43, 41, 30, 30, 27, and 25; (bottom) 23, 25, 25, 32, 20, 29, 24, 28, 27, and 17.p values of statistical test between each strain and control JN1601 in ATR conditions were as follows: top, $6.67 \times 10^{-9}, 2.25 \times 10^{-2}, 1.00,1.63 \times 10^{-3}$; bottom, 1.00, 0.319, 0.321, 4.82 $\times 10^{-4}\left({ }^{* * *} p<0.001{ }^{* *} p<0.01,{ }^{*} p<0.05\right.$, Dunnett's test). c, Curving induced by the phasic activation of downstream interneurons after preculture at different NaCl concentrations. Strains: AIY-ChR2, SRS281; AIA-ChR2, JN1605; AIB-ChR2, SRS291; AIA/AIB-ChR2, JN1608. Numbers of animals for each bar: AIY-ChR2, 25, 28, 30, 23, 30, and 25; AIA-ChR2, 33, 34, 23, 30, 36, and 30; AIB-ChR2, 32, 30, 24, 28, 26, and 28; AIA/AIB-ChR2, 27, 30, 38, 44,24 , and 17. The effects of concentration of preculture on curving bias were not significant in all strains: AlY-ChR2, $F_{(2,155)}=0.615, p=0.542 ; \mathrm{AlA}-\mathrm{ChR2}, F_{(2,180)}=0.203, p=0.816 ; \mathrm{AlB}-\mathrm{ChR2}$, $F_{(2,162)}=0.744, p=0.477 ;$ AIA/AIB-ChR2, $F_{(2,174)}=1.09, p=0.337 ;$ and the effects of the presence of ATR on curving bias were significant in AIY-ChR2 $\left(F_{(1,155)}=189, p<2 \times 10-16\right)$ and AIA-ChR2 $\left(F_{(1,180)}=12.1, p=6.40 \times 10^{-4}\right)$, but were not significant in AIB-ChR2 $\left(F_{(1,162)}=0.435, p=0.511\right)$ and AIA/AIB-ChR2 $\left(F_{(1,174)}=0.518, p=0.473,2\right.$-way ANOVA). $p$ values of statistical tests between ATR and No ATR in each preculture condition in AIY-ChR2 are $1.00 \times 10^{-11}, 2.02 \times 10^{-7}$, and $3.15 \times 10^{-13}$. $p$ values of statistical tests between ATR and No ATR in each preculture condition in AIA-ChR2 are $3.86 \times 10^{-2}, 5.66 \times 10^{-3}$, and 0.372 (Student's t test). d, A possible neural mechanism that generates bidirectional klinotaxis in salt chemotaxis. Information from the ASER salt-sensing neurons is mediated by AIY neurons and other unknown pathways.

\section{Discussion}

The neural basis of chemotaxis in C. elegans has been studied for years. As sensory inputs, the responses of neurons to chemical cues, such as odor and salts, have been well described (Chalasani et al., 2007; Suzuki et al., 2008). As behavioral outputs, two major strategies for chemotaxis are known: (1) klinokinesis, in which the animal changes the frequency of a complex turning behavior that consists of consecutive short reversals and omega turns, and (2) klinotaxis, in which the animal gradually curves during forward locomotion in response to the chemical gradient perpendicular to the body axis (Pierce-Shimomura et al., 1999; Iino and Yoshida, 2009).

Recent studies on chemotaxis to odor have provided a better understanding of the neural mechanisms that connect these sen- 
sory inputs to behavioral outputs. AWC chemosensory neurons show calcium responses to the removal of odor, such as isoamyl alcohol or butanone (Chalasani et al., 2007). Three major interneuron classes that are postsynaptic to AWC neurons are also responsive to odor; namely, AIB neurons respond to odor removal, while AIA and AIY neurons respond to odor exposure (Chalasani et al., 2007, 2010). At the behavioral level, the ablation of AIB neurons decreases reversal events, and the ablation of AIA or AIY neurons increases reversal events (Wakabayashi et al., 2004; Gray et al., 2005). Through experiments to control activity of the $\mathrm{AWC}^{\mathrm{ON}}$ neuron or of postsynaptic interneurons, it has been suggested that AIB interneurons drive klinokinesis while AIY interneurons drive klinotaxis, probably in response to odorevoked activity changes of AWC neurons (Kocabas et al., 2012).

These three interneuron classes, AIA, AIB, and AIY, are postsynaptic to many sensory neurons and are likely to also be involved in the chemotaxis to other chemicals. For the chemotaxis to $\mathrm{NaCl}, \mathrm{ASE}$ neurons are essential in the low concentration range $(<200 \mathrm{~mm}$; Hukema et al., 2008). The first-layer interneuron classes that are postsynaptic to ASE neurons are AIA, AIB, and AIY, the same set as for AWC neurons (Fig. 3a; White et al., 1986). Chemotaxis to odor is similar to chemotaxis to $\mathrm{NaCl}$ except that chemotaxis to $\mathrm{NaCl}$, unlike chemotaxis to odor, will change according to experience (Saeki et al., 2001; Kunitomo et al., 2013). Experience also affects the underlying klinotaxis behavior. This plasticity requires the ASER neuron, which is one of a bilateral ASE chemosensory neuron pair (Tomioka et al., 2006; Kunitomo et al., 2013). In this study, we performed ASER activation experiments in animals that experienced different $\mathrm{NaCl}$ concentrations and found that input from a single chemosensory ASER neuron was sufficient to drive both ipsilateral and contralateral curving outputs that corresponded to negative and positive klinotaxis to $\mathrm{NaCl}$, respectively (Fig. $2 b$ ). The plastic curving outputs that depended on previous experiences were consistent with the plastic klinotaxis to $\mathrm{NaCl}$ (Kunitomo et al., 2013). With ASER activation in interneuron-ablated animals, we found that AIY interneurons are required only for ipsilateral curving caused by ASER activation but not for contralateral curving (Fig. 3b). Consistently, direct activation of interneurons showed that AIY can drive ipsilateral curving but not contralateral curving (Fig. $3 c$ ). Therefore, it is deduced that the plastic bidirectional klinotaxis to $\mathrm{NaCl}$ is regulated by switching between microcircuits that contain AIY and those that do not contain AIY (Fig. 3d). Double ablation of AIA and AIB interneurons suggested that these neurons redundantly regulate curving contralateral to the side of ASER activation, or positive klinotaxis to $\mathrm{NaCl}$ (Fig. $3 b$ ). However, simultaneous activation of AIA and AIB neurons did not induce any biased curving (Fig. 3c). One possibility is that AIA and AIB neurons regulate positive klinotaxis only when activated with a time lag. Therefore, simultaneous activation of both neurons was not sufficient for curving.

Note that in interneuron activation experiments both left and right bilateral cells of each interneuron class (AIYL and AIYR, AIAL and AIAR, AIBL and AIBR) were activated at the same time because technical limitations made it impossible to genetically or spatially segregate the left and right neurons. There is strong leftright asymmetry in the connectivity from ASE chemosensory neurons to downstream interneurons: the right ASE neuron is strongly connected to the right members of AIY and AIB neurons (ASER to AIYR/AIBR), which is also true for the left members (ASEL to AIYL/AIBL). There is also a clear functional left-right asymmetry of ASE neurons in sensing $\mathrm{NaCl}$ concentration changes: the ASEL neuron responds to increase in $\mathrm{NaCl}$ concen- tration while the ASER neuron responds to decrease of $\mathrm{NaCl}$ (Suzuki et al., 2008). Therefore, there remains a possibility that activating both left and right members of interneurons simultaneously might not precisely mimic the phasic $\mathrm{NaCl}$ concentration change in some cases.

Considering that both $\mathrm{AWC}{ }^{\mathrm{ON}}$ and ASER neurons are stimulated by the removal of responsive chemicals (Chalasani et al., 2007; Suzuki et al., 2008; Kunitomo et al., 2013), it is possible that the contralateral and ipsilateral curving induced by $\mathrm{AWC}^{\mathrm{ON}} \mathrm{ac}-$ tivation might be differently mediated by AIY neurons.

The neural pathways for contralateral curving to ASER activation (or positive klinotaxis to $\mathrm{NaCl}$ ) are still unknown. Ablation of each of the first-layer interneurons that were postsynaptic to the ASER neuron did not abolish the contralateral curving in response to the phasic activation of the ASER neuron (Fig. $3 a, b$ ). There might be other functional pathways from the ASER neuron through other sensory neurons that mediate contralateral curving. Another possibility is that there is an extensive functional redundancy between the pathways in which these interneurons transmit the ASER activation pattern. A defect in the contralateral curving observed in the AIA/AIB double-ablation animals at least partially supports the latter possibility.

\section{References}

Bargmann CI, Horvitz HR (1991) Chemosensory neurons with overlapping functions direct chemotaxis to multiple chemicals in C. elegans. Neuron 7:729-742. CrossRef Medline

Brenner S (1974) The genetics of Caenorhabditis elegans. Genetics 77:71-94. Medline

Chalasani SH, Chronis N, Tsunozaki M, Gray JM, Ramot D, Goodman MB, Bargmann CI (2007) Dissecting a circuit for olfactory behaviour in Caenorhabditis elegans. Nature 450:63-70. CrossRef Medline

Chalasani SH, Kato S, Albrecht DR, Nakagawa T, Abbott LF, Bargmann CI (2010) Neuropeptide feedback modifies odor-evoked dynamics in Caenorhabditis elegans olfactory neurons. Nat Neurosci 13:615-621. CrossRef Medline

Edwards SL, Charlie NK, Milfort MC, Brown BS, Gravlin CN, Knecht JE, Miller KG (2008) A novel molecular solution for ultraviolet light detection in Caenorhabditis elegans. PLoS Biol 6:e198. CrossRef Medline

Granato M, Schnabel H, Schnabel R (1994) pha-1, a selectable marker for gene transfer in C. elegans. Nucleic Acids Res 22:1762-1763. CrossRef Medline

Gray JM, Hill JJ, Bargmann CI (2005) A circuit for navigation in Caenorhabditis elegans. Proc Natl Acad Sci U S A 102:3184-3191. CrossRef Medline

Hukema RK, Rademakers S, Jansen G (2008) Gustatory plasticity in C. elegans involves integration of negative cues and $\mathrm{NaCl}$ taste mediated by serotonin, dopamine, and glutamate. Learn Mem 15:829-836. CrossRef Medline

Iino Y, Yoshida K (2009) Parallel use of two behavioral mechanisms for chemotaxis in Caenorhabditis elegans. J Neurosci 29:5370-5380. CrossRef Medline

Izquierdo EJ, Lockery SR (2010) Evolution and analysis of minimal neural circuits for klinotaxis in Caenorhabditis elegans. J Neurosci 30:1290812917. CrossRef Medline

Kocabas A, Shen CH, Guo ZV, Ramanathan S (2012) Controlling interneuron activity in Caenorhabditis elegans to evoke chemotactic behaviour. Nature 490:273-277. CrossRef Medline

Kunitomo H, Sato H, Iwata R, Satoh Y, Ohno H, Yamada K, Iino Y (2013) Concentration memory-dependent synaptic plasticity of a taste circuit regulates salt concentration chemotaxis in Caenorhabditis elegans. Nat Commun 4:2210. CrossRef Medline

Lin CH, Tomioka M, Pereira S, Sellings L, Iino Y, van der Kooy D (2010) Insulin signaling plays a dual role in Caenorhabditis elegans memory acquisition and memory retrieval. J Neurosci 30:8001-8011. CrossRef Medline

Liu J, Ward A, Gao J, Dong Y, Nishio N, Inada H, Kang L, Yu Y, Ma D, Xu T, Mori I, Xie Z, Xu XZ (2010) C. elegans phototransduction requires a G protein-dependent cGMP pathway and a taste receptor homolog. Nat Neurosci 13:715-722. CrossRef Medline 
Matsuki M, Kunitomo H, Iino Y (2006) Goalpha regulates olfactory adaptation by antagonizing Gqalpha-DAG signaling in Caenorhabditis elegans. Proc Natl Acad Sci U S A 103:1112-1117. CrossRef Medline

Mello CC, Kramer JM, Stinchcomb D, Ambros V (1991) Efficient gene transfer in C. elegans: extrachromosomal maintenance and integration of transforming sequences. EMBO J 10:3959-3970. Medline

Pierce-Shimomura JT, Morse TM, Lockery SR (1999) The fundamental role of pirouettes in Caenorhabditis elegans chemotaxis. J Neurosci 19:95579569. Medline

Saeki S, Yamamoto M, Iino Y (2001) Plasticity of chemotaxis revealed by paired presentation of a chemoattractant and starvation in the nematode Caenorhabditis elegans. J Exp Biol 204:1757-1764. Medline

Suzuki H, Thiele TR, Faumont S, Ezcurra M, Lockery SR, Schafer WR (2008) Functional asymmetry in Caenorhabditis elegans taste neurons and its computational role in chemotaxis. Nature 454:114-117. CrossRef Medline
Tomioka M, Adachi T, Suzuki H, Kunitomo H, Schafer WR, Iino Y (2006) The insulin/PI 3-kinase pathway regulates salt chemotaxis learning in Caenorhabditis elegans. Neuron 51:613-625. CrossRef Medline

Wakabayashi T, Kitagawa I, Shingai R (2004) Neurons regulating the duration of forward locomotion in Caenorhabditis elegans. Neurosci Res 50: 103-111. CrossRef Medline

Ward S (1973) Chemotaxis by the nematode Caenorhabditis elegans: identification of attractants and analysis of the response by use of mutants. Proc Natl Acad Sci U S A 70:817-821. CrossRef Medline

White JG, Southgate E, Thomson JN, Brenner S (1986) The structure of the nervous system of the nematode Caenorhabditis elegans. Philos Trans $\mathrm{R}$ Soc Lond B Biol Sci 314:1-340. CrossRef Medline

Yoshida K, Hirotsu T, Tagawa T, Oda S, Wakabayashi T, Iino Y, Ishihara T (2012) Odour concentration-dependent olfactory preference change in C. elegans. Nat Commun 3:739. CrossRef Medline 